Original Contribution

\title{
THE ROLE OF PSYCHOSOCIAL INTERVENTION ON THE COURSE OF THE DISEASE IN PERSONS WITH SEVERE MENTAL DISORDERS
}

\author{
A. Popova-Varbanova*, V. Getova \\ Department of Social Activities, Faculty of Medicine, Trakia University, Stara Zagora, Bulgaria
}

\begin{abstract}
The study includes 220 people with severe mental disorders, men and women, of ages 18-70, placed for compulsory treatment in psychiatric institutions in the area of Sofia city and Sofia region. The patients were hospitalized for the period from January 2013 to December 2015. Social factors, such as: age, family status and support were taken into account. The patients' usages of the service of psychosocial rehabilitation, as well as subsequent hospitalizations, were monitored for one year.
\end{abstract}

Key words: Social factors, Resocialization, The impact of disease damage, The care programs, Rehospitalization

\section{INTRODUCTION}

New technologies for treatment of mental disorders are much broader than pure medical intervention which, in psychiatry, consists mostly of therapy using drugs and some nonpharmacological methods such as transcranial magnetic electrostimulation and electroconvulsive therapy. They rely on organized groups of specialists like doctors, nurses, psychologists, social workers and go as far as employing new positions and even professions like case leader and etc. (1).The training of social skills can't be clinically effective when there are no conditions for interpersonal and material reinforcements. Let's take, for example, a patient with schizophrenia, who lives on a social disability pension, isolated, in subsidized apartment, built by a project for cheap apartments for people in need without relatives and friends, and he doesn't take part in daily treatment programs. In this case there won't be any use of training in social skills, unless his surrounding environment changes and becomes more rewarding. Similarly, a patient in a chronic ward could benefit from treatment in social skills only if there are enough rewarding interactions with the other patients and the staff. (2). In the 90s, the concept of

*Correspondence to: Popova - Varbanova A., Trakia University, Faculty of Medicine,

Department of Social Activities, 11 "Armeiska" street, 6000, Stara Zagora, Bulgaria, telephone: 042 644-417, e-mail:dr.anapopova@gmail.com recovery from severe mental illness becomes established as a leading paradigm in psychosocial rehabilitation. (3). It is a natural result of the processes of deinstitutionalization and is based on data collected from long-term monitoring of schizophrenic patients from different populations, which indicate considerable share of spontaneous improvement in later stages of the condition. The rehabilitation of people with mental disorders is an important part of the complex of restorative actions, and alongside treatment and the care programs, it's directed toward reducing the impact of disease damage.

\section{TASKS AND ASSUMPTIONS OF THE RESEARCH}

The study is based on people with severe mental disorders during psychosocial intervention. The current study is covering the cases of 220 people with severe mental disorders, classified by International Classification of Diseases with the following diagnoses: F 06.2, F 06.3, F 10.51, F 11.5, F 20.0, F 20.6, F 22.0, F 23.1, F 25.2, F 30.2, F 31.6, F 32.1, F 33.2. The age of the patients in the study is between 18 and 71 . Their family status and support are the main criteria. The patients were having their compulsory treatment in psychiatric facilities in Sofia city and Sofia region, namely

\footnotetext{
- MHC "Prof. N. Shipkovenski" LTD

- SPH "St. Ivan Rilski”"

- SPH Karlukovo
} 
- MHC bul. Slivnitsa

- Military Medica Psychiatric clinic

- UMBAL Aleksandrovska EAD

- MHATNP St. Naum

- $\quad$ Outside the region of Sofia

Within the period from 2013 to 2015 , their use of the service of psychosocial rehabilitation and compulsory treatment has been monitored.

\section{STUDY HYPOTHESIS}

First of all, there is interdependence between the number of re-hospitalizations and the number of patients with severe mental disorders. Secondly, it is assumed that there is a difference in the number of re-hospitalization compared to such factors as: diagnosis, age, marital status and support and conducted psychosocial rehabilitation.

\section{METHODOLOGY}

The analysis was based on data collected from the medical reports of patients. The SPSS software was used for statistical processing of the collected data. The following methods (assays) are used in this study:

- descriptive statistics, frequency analysis;

- analysis of variance;

- correlation analysis;

\section{RESULTS AND INTERPRETATION}

There are psychosocial and demographic factors for patients with mental disorder, which will be observed below. Some of them may affect the treatment of the patients.

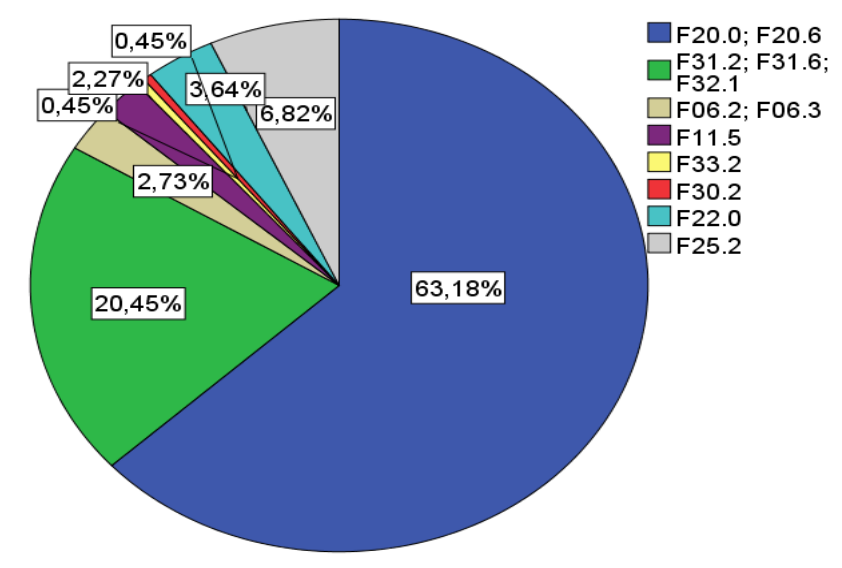

Figure 1. Distribution of examined patients in diagnoses (in percentage)

As we see in the Figure 1, 63.18\% of the people in the study have the diagnose F20.0 (Paranoid schizophrenia) and F20.6 (simple schizophrenia), $20.45 \%$ of the people have diagnoses F31.2 (Bipolar Affective Disorder current episode manic with psychotic symptoms), F31.6 (Current episode -Mixtures), F32.1 (Bipolar Affective Disorder current moderate depressive episode), $2.73 \%$ of the sample have diagnoses F06.2 and F06.3,
$2.27 \%$ are with $\mathrm{F} 11.5,0.45 \%$ are with $\mathrm{F} 30.2$ (Mania with psychotic disorders) and F33.2. The share of F22.0 (Persistent delusional disorder) is $3.64 \%$ and F25.2 (Schizoaffective disorder. Mixed) is $6.82 \%$.

In Figure 2 we can see that the bigger part $(53.18 \%)$ of the examined patients with severe mental disorders weren't re-hospitalized.

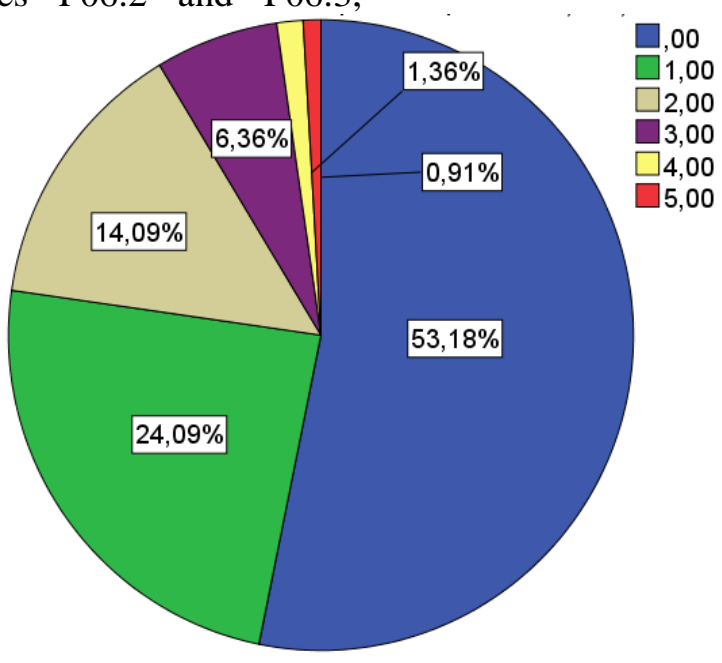

Figure 2. Distribution of the examined patients according to the number of re-hospitalization (in percentage) 
- One re-hospitalization $-24.09 \%$

- Two re-hospitalizations $-14.09 \%$

- Three re-hospitalizations $-6.36 \%$

- Four re-hospitalizations $-1.36 \%$

- Five re-hospitalizations $-0.91 \%$

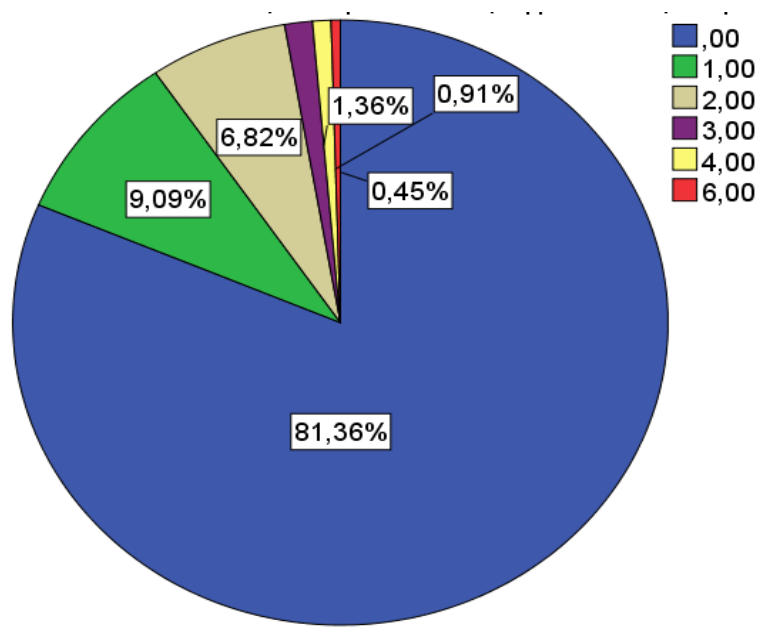

Figure 3. Distribution of the examined patients according to their participation in psychosocial rehabilitation (in percentage)

In Figure 3 we can see that $81.36 \%$ of the examined patients didn`t visit day hospital. Day hospital is a unit of semi-stationary psychiatric care in the system of psychiatric hospitals. This kind of unit helps patients with mental disorders by doing diagnoses, treatment, observation, expert activity and resocialization of people. We can also see in
Figure 3 that $9.09 \%$ of the patients were visiting psychosocial rehabilitation once, $6.82 \%$ - twice, $1.36 \%$ - three times, $0.91 \%$ four times and $0.45 \%$ - five times. Inclusion of people in psychosocial rehabilitation in Bulgaria is still voluntary, according to current legislation. That explains these results.

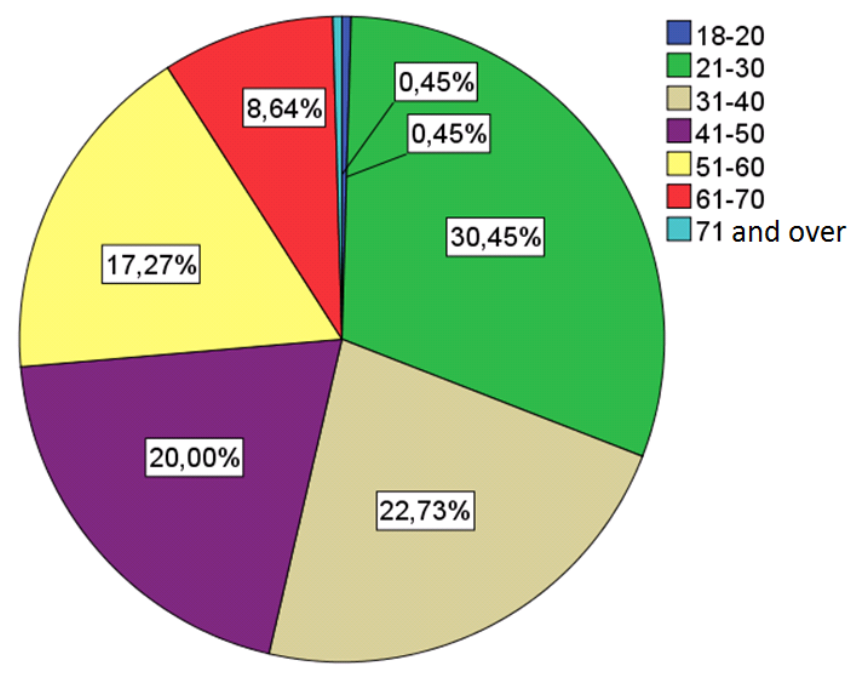

Figure 4. Distribution of examined patients by age (in percentages)

As we can see in Figure 4, one third (30.45\%) of the people with severe mental disorders are aged $21-30,22.73 \%$ are $31-40$ years old, $20 \%$ are 41-50 years old, $17.27 \%$ - 51-60 years old, $8.64 \%$ are $61-70$ years old. The people aged $18-20$ and $71+$ have equal shares $(0.45 \%)$.
As we see in Figure 5 most of the people in the study $(69.55 \%)$ were unmarried, $15.45 \%$ divorced, $12.27 \%$ - married and $2.73 \%$ widow/widower. Family status is very important for the affected. Family support serves as an important psychotherapeutic resource. For that reason, it is included for consideration in this study. 


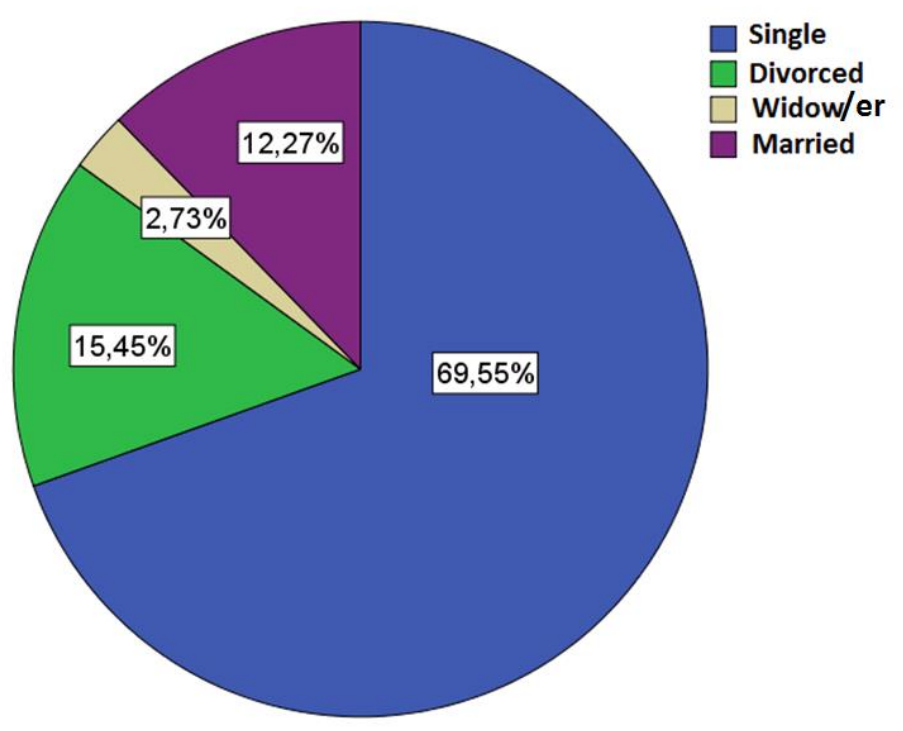

Figure 5. Distribution of examined potions by family status (in percentage)

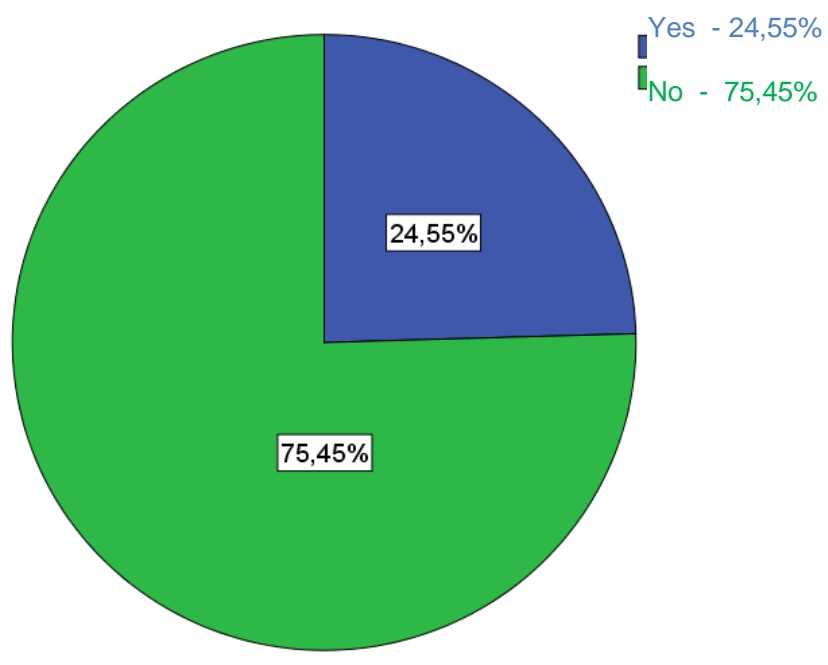

Figure 6. Distribution of the examined patients according to whether they have declared family support or not (in percentage)

As we can see in fig. 6 most of the patients $(75.45 \%)$ didn`t get family support and only
$24.55 \%$ were supported by their family. This support is very important for the patients' psychological recovery.

Table 1. Statistics of criterion ${ }^{a, b}$

\begin{tabular}{|c|c|}
\cline { 2 - 2 } \multicolumn{1}{c|}{} & Re-hospitalization 3 years / 2013,2014,2015/ \\
\hline Chi-Square & 2,128 \\
\hline $\mathrm{df}$ & 3 \\
\hline Asymp. Sig. &, 546 \\
\hline
\end{tabular}

a. Kruskal Wallis Test

b. Grouping variable: Family status

The result in Table 1 shows that there are no statistically significant $(\mathrm{p}=0,546)$ differences between re-hospitalization and the family status of patients with severe mental disorders. 
Table 2. Ranks

\begin{tabular}{|l|l|l|l|}
\cline { 2 - 4 } \multicolumn{1}{c|}{} & $\begin{array}{l}\text { Psychosocial rehabilitation / } \\
\text { Day hospital }\end{array}$ & № & Medium rank \\
\hline \multirow{4}{*}{$\begin{array}{l}\text { Re-hospitalizations for 3 years. / } \\
2013,2014,2015 /\end{array}$} & 0 & 179 & 105,33 \\
\cline { 2 - 4 } & 1 & 20 & 119,95 \\
\cline { 2 - 4 } & 2 & 15 & 158,90 \\
\cline { 2 - 4 } & 3 & 3 & 87,33 \\
\cline { 2 - 4 } & 4 & 2 & 101,50 \\
\hline & 6 & 208,50 \\
\cline { 2 - 4 } & Total & 220 & \\
\hline
\end{tabular}

As we see in Tables 2 and 3 there is a statistically significant difference $(p=0,008)$ between the number of re-hospitalizations and the number of conducted psychosocial rehabilitations. The lowest count is for patients with re-hospitalization after three visits to the daily hospital (average rank is 87.33). The largest number of rehospitalizations is for patients with 6 visits to psychosocial rehabilitation.

\section{CONCLUSION}

The results obtained using variance analysis support the hypothesis that there is a difference between the number of rehospitalizations and the number of conducted psychosocial rehabilitations (Tables 2 and 3). The assumption that there is a difference in the number of rehospitalizations due to factors such as disability, age, diagnosis, education, family status, has not been confirmed in the present study, which can only emphasize the significance and importance of the psychosocial rehabilitation.

Table 3. Statistics of criterion ${ }^{a, b}$

\begin{tabular}{|l|l|}
\hline & Re-hospitalizations in 3 years / 2013,2014,2015/ \\
\hline Chi-Square & 15,737 \\
\hline Df & 5 \\
\hline Asymp. Sig. & $\mathbf{, 0 0 8}$ \\
\hline
\end{tabular}

a. Kruskal Wallis Test

b. Grouping variable: Psychosocial rehabilitation / Daily hospital

\section{REFERENCES}

1. Castle, D.J., Buckley P. F., (2012), schizophrenia, Oxford University Press

2. Marinov, P. Schizophrenia (2010), "ASSIA

- Rosen Mladenov," Sofia
3. Kaplan, H., Sadock, B., Grebb, J. (1994) Synopsis of Psychiatry, Williams \& Wilkins 